\title{
JEAN-PIERRE FRANÇOISE
} MOHAND SMAÏLI

\section{Lemme de Morse transverse pour des puissances de formes de volume}

Annales de la faculté des sciences de Toulouse $6^{e}$ série, tome $3, \mathrm{n}^{\circ} 1$ (1994), p. 81-89

<http://www.numdam.org/item?id=AFST_1994_6_3_1_81_0>

(C) Université Paul Sabatier, 1994, tous droits réservés.

L'accès aux archives de la revue «Annales de la faculté des sciences de Toulouse » (http://picard.ups-tlse.fr/ annales/) implique l'accord avec les conditions générales d'utilisation (http://www.numdam.org/conditions). Toute utilisation commerciale ou impression systématique est constitutive d'une infraction pénale. Toute copie ou impression de ce fichier doit contenir la présente mention de copyright.

\section{NumDam}

Article numérisé dans le cadre du programme

Numérisation de documents anciens mathématiques

http://www.numdam.org/ 


\title{
Lemme de Morse transverse pour des puissances de formes de volume $e^{(*)}$
}

\author{
Jean-Pierre Françoise et Mohand Smaïli ${ }^{(1)}$
}

RÉSUMÉ. - On considère l'ensemble des "puissances de forme volume"

$$
f(x, y)^{\alpha} h(x) \mathrm{d} x,
$$

où $f(x, y)$ est un germe de Morse transversalement au feuilletage $p$ : $(x, y) \rightarrow y$. On fait opérer sur cet ensemble le groupe des germes de difféomorphismes $\operatorname{Diff}\left(\mathbb{R}^{2 n}, 0\right)$. On démontre qu'il existe toujours un élément de la forme $\left(\Sigma_{i} a_{i}(y) x_{i}^{2}\right)^{\alpha} \mathrm{d} x$ dans une orbite de l'action. On termine en donnant une application au calcul d'intégrales de la phase stationnaire.

Abstract. - We consider the set of powers of volume forms

$$
f(x, y)^{\alpha} h(x) \mathrm{d} x
$$

where $f: \mathbb{R}^{2 n}, 0 \rightarrow \mathbb{R}, 0$ is a germ of function which is transversally of Morse type relatively to the foliation $p:(x, y) \rightarrow y$. There is a normal form to the action of $\operatorname{Diff}\left(\mathbb{R}^{2 n}, 0\right)$ on each orbits which is of the type: $\left(\Sigma_{i} a_{i}(y) x_{i}^{2}\right)^{\alpha} \mathrm{d} x$. We conclude with an application to the computation of integrals of the stationnary phase.

\section{Introduction}

Rappelons le résultat obtenu par J. Vey [V] en 1978 :

Soit $f$ un germe de fonction analytique en $0 \in \mathbb{R}^{n}$ telle que $f(0)=0$ et $\delta f(0)=0$. On suppose que $f$ présente une singularité du type de Morse en 0 , et on désigne par $Q$ la forme quadratique à laquelle $f$ est tangente

(*) Reçu le 26 mars 1993

(1) Université de Paris VI, U.F.R. 920, Mathématiques, U.R.A. 213, Couloir 46-56, 5ème étage, 4 place Jussieu, F-75252 Paris (France) 
à l'ordre trois en 0 . Soit d'autre part $\omega$ un germe de forme volume en 0 . In existe un changement local de coordonnées qui conserve le volume $\omega$ et qui ramène la fonction $f$ à une fonction $S(Q)$. La série entière $t \rightarrow S(t)$ est caractéristique du couple $(f, \omega)$ et est reliée par une équation différentielle ordinaire à la fonction $t \rightarrow \ell(t)=\int_{f \leq t} \omega$, dans le cas où $Q$ est définie.

Cet énoncé a été par la suite étendu au cas différentiable par V. Guillemin [G] et indépendamment par Y. Colin de Verdière et J. Vey [CV-V]. La signification géométrique en est parfaitement claire : alors qu'on peut conjuguer un germe de fonction qui présente une singularité de Morse à sa hessienne par un difféomorphisme quelconque, on ne peut le faire par un difféomorphisme isochore (c'est-à-dire qui conserve le volume) et l'unique obstruction à le faire est représentée par l'intégrale qui détermine la classe de cohomologie relative de $\omega$ par rapport à $f$, dans le cas où $Q$ est définie.

On pourra se reporter à $[F]$ et à la thèse de $C$. Roche $[R]$ pour une extension aux singularités quelconques.

Arnol'd [A] a introduit la notion de puissance de forme volume $f(\mathrm{~d} x)^{\beta}$ ( $\beta$ est un paramètre complexe) où $f$ est un germe de fonction analytique (resp. $C^{\infty}$ ) à singularité isolée à l'origine. $\mathrm{I}$ revient au même de considérer les objets $f^{\alpha}(\mathrm{d} x)(\alpha=1 / \beta)$.

Pour $\beta=1$, on obtient les formes volumes usuelles, pour $\beta=-1$, les champs de vecteurs si $n=1$, les structures de Poisson si $n=2$. Le cas $\beta=1 / 2$ est particulièrement intéressant et correspond aux demidensités utilisées par Guillemin et Sternberg [G-S] pour la quantification géométrique.

On pourra consulter $[\mathrm{V}],[\mathrm{L}]$ et $[\mathrm{K}-\mathrm{L}]$ pour des développements ultérieurs du sujet.

Soit $f$ un germe (analytique, différentiable) de fonction de $\mathbf{R}^{2 n}, 0 \rightarrow \mathbb{R}, 0$ et

$$
p: \mathbf{R}^{2 n}, 0 \longrightarrow \mathbb{R}^{n}, \mathbf{0}, \quad p:(x, y) \longrightarrow y
$$

un feuilletage local en $\mathbf{0}$.

On suppose que $f$ présente un point de Morse en restriction à la feuille $p^{-1}(p(0))$ (et donc sur les feuilles voisines) en sorte que :

i) $f=\Sigma_{i} a_{i}(y) x_{i}^{2}+R(x, y)$;

ii) $\operatorname{det} \frac{\delta a_{i}}{\delta y_{j}}(0)=0$;

iii) $R(x, y)$ est nul à l'ordre trois le long de $x=0$. 
Considérons l'ensemble des

$$
f(x, y)^{\alpha} h(x) \mathrm{d} x, \quad h(0)=1,
$$

sur lequel on fait opérer $\operatorname{Diff}\left(R^{2 n}, 0\right)$ de la manière suivante :

$$
\begin{aligned}
\Phi & =\left(x^{\prime}=x^{\prime}(x, y), y^{\prime}=y^{\prime}(x, y)\right), \\
\Phi^{*} f(x, y)^{\alpha} h(x) \mathrm{d} x & =f\left(x^{\prime}(x, y), y^{\prime}(x, y)\right)^{\alpha} h\left(x^{\prime}\right) \mathrm{Jac}\left(x / x^{\prime}\right) \mathrm{d} x .
\end{aligned}
$$

Nous démontrons ici le résultat.

THÉORÈME .- Il existe un représentant de l'orbite de $f(x, y)^{\alpha} h(x) \mathrm{d} x$ sous l'action de $\operatorname{Diff}\left(\mathbf{R}^{2 n}, 0\right)\left(C^{\infty}, C^{\omega}\right)$ de la forme $\left(\Sigma_{i} a_{i}(y) x_{i}^{2}\right)^{\alpha} \mathrm{d} x$.

Une des motivations de ce théorème est l'étude de certaines intégrales de la phase stationnaire qui dépendent de paramètres. Nous en donnons un exemple à la fin de cet article.

Pour fixer les notations, nous supposons que

$$
a_{i}(0)>0, \quad \text { pour } i=1, \ldots, p
$$

et

$$
a_{i}(0)<0, \quad \text { pour } i=p+1, \ldots, p+q=m .
$$

Il sera commode, dans la suite, de se référer à la régularité $\left(C^{\infty}, C^{\omega}\right)$ d'une donnée (fonction, forme différentielle, champ de vecteurs) suivant qu'elle est différentiable ou analytique. L'expression "germe de" sera omise mais sous-entendue.

\section{Le lemme de Morse isochore à paramètres}

THÉORÈME 1. - Soit $f(\lambda, x)$ une fonction $\left(C^{\infty}, C^{\omega}\right)$ en la variable $x$ et le paramètre $\lambda$ et qui présente une singularité de Morse en $x=0$. On suppose, pour fixer les notations, que $f(\lambda, 0)=0$ et

$$
Q=\operatorname{Hess}_{0}(f)=\Sigma_{i} a_{i}(\lambda) x_{i}^{2} .
$$

Il existe un changement de variable $\left(C^{\infty}, C^{\omega}\right) x^{\prime}=x^{\prime}(x, \lambda)$ qui dépend $\left(C^{\infty}, C^{\omega}\right)$ de $\lambda$, qui conserve la forme $\omega$ et conjugue $f \grave{a} F(\lambda, Q(x, \lambda))$, où

$$
Q(x, \lambda)=\operatorname{Hess}_{0}(f)=\Sigma_{i} a_{i}(\lambda) x_{i}^{2} .
$$


Dans le cas analytique, on peut écrire $F(\lambda, Q(x, \lambda))$ comme une série convergente :

$$
\Sigma_{k} c_{k}(\lambda) Q(x, \lambda)^{k}
$$

où les $c_{k}(\lambda)$ sont des fonctions analytiques de $\lambda$.

\section{Démonstration}

Première étape : le lemme de Morse à paramètres

Il faut commencer par revenir au lemme de Morse à paramètres luimême. Au lieu de fournir une référence, nous préférons rétablir rapidement une preuve à partir de la méthode des chemins.

Écrivons :

$$
f(\lambda, x)=\Sigma_{i} a_{i}(\lambda) x_{i}^{2}+R(x, \lambda),
$$

Puis introduisons

$$
f_{t}(x, \lambda)=\Sigma_{i} a_{i}(\lambda) x_{i}^{2}+t R(x, \lambda), \quad t \in[0,1]
$$

et

$$
R(x, \lambda)=\Sigma_{i} R_{i}(x, \lambda) x_{i}=\langle R, x\rangle .
$$

Soit $\delta f_{t} / \delta x$ le vecteur de composantes $\delta f_{t} / \delta x_{i}$

$$
\frac{\delta f_{t}}{\delta x_{i}}=2 a_{i}(\lambda) x_{i}+t \frac{\delta R}{\delta x_{i}}=\Sigma_{j}\left(2 a_{i}(\lambda) \delta_{i j}+t A_{i j}\right) x_{j}
$$

pour une certaine matrice $A(x, \lambda)$ qui est $\left(C^{\infty}, C^{\omega}\right)$ en $\lambda$.

Soit $B^{-1}(t, x, \lambda)$ la matrice inverse de $B$ définie par

$$
B_{i j}=2 a_{i}(\lambda) \delta_{i j}+t A_{i j} .
$$

On a donc l'égalité vectorielle :

$$
x=B^{-1} \frac{\delta f_{t}}{\delta x}
$$

et

$$
f_{t}=\left\langle R, B^{-1} \frac{\delta f_{t}}{\delta x}\right\rangle .
$$

Soit $X_{t}(x, \lambda)$ le vecteur défini par

$$
\begin{gathered}
X_{t}(x, \lambda)=\Sigma_{i, j} R_{i} B_{i j}^{-1}(x, \lambda) \frac{\delta}{\delta x_{j}}, \\
-84-
\end{gathered}
$$


nous obtenons que

$$
f_{t}=X_{t}(x, \lambda) \cdot f_{t} .
$$

Le champ $X_{t}(x, \lambda)$ est $\left(C^{\infty}, C^{\omega}\right)$ en $(x, \lambda)$, et $X_{t}(0, \lambda)=0$. П existe donc un voisinage compact $B(0, r)$ dans l'espace des $\lambda$ et un voisinage dans l'espace des $x$ tel que le flot de $X_{t}(x, \lambda)$ existe pour $t \in[0,1]$ sur $B(0, r)$ et est $\left(C^{\infty}, C^{\omega}\right)$ en $\lambda$.

Rappelons que le fait que $X_{t}(x, \lambda)$ s'annule en $x=0$ est essentiel pour que le flot existe pour toute valeur $t \in[0,1]$. La régularité $\left(C^{\infty}, C^{\omega}\right)$ relative à $\lambda$ est une conséquence des théorèmes classiques sur la régularité des solutions des équations différentielles comme fonctions d'un paramètre.

Terminons l'argument : $F_{1}(x, \lambda)$ est un difféomorphisme qui dépend $\left(C^{\infty}, C^{\omega}\right)$ de $\lambda$ et qui ramène $f$ à la hessienne

$$
Q(x, \lambda)=\Sigma_{i} a_{i}(\lambda) x_{i}^{2} .
$$

La forme volume $\omega$ est transformée par $F_{1}$ en une forme

$$
B(x, \lambda) \mathrm{d} x_{1} \wedge \ldots \wedge \mathrm{d} x_{n}
$$

où $B$ dépend $\left(C^{\infty}, C^{\omega}\right)$ de $x$ et du paramètre $\lambda$.

Deuxième étape : la moyennisation dans le groupe compact $K=\operatorname{SO}(p) \times$ $\mathrm{SO}(q)$

On reprend ici une version à paramètre d'un passage de l'article de Colin de Verdière et Vey [CV-V] ou celui de Guillemin [G].

Soit $g$ un élément du groupe $K$. Supposons dans un premier temps qu'il est proche de l'identité et que on l'interpôle par un groupe à un paramètre $g_{t}$. Soit $X_{t}$ l'arc de champ de vecteur sur le groupe qui engendre le groupe à un paramètre.

On peut écrire :

$$
\begin{aligned}
g_{1}^{*}(\omega)-\omega & =\int_{[0,1]} \frac{\mathrm{d}}{\mathrm{d} t}\left(g_{t}^{*}(\omega)\right) \mathrm{d} t \\
& =\int_{[0,1]} \mathrm{d} g_{t}^{*}\left(i_{X} \omega\right) \mathrm{d} t .
\end{aligned}
$$

Comme $\mathrm{d} Q \wedge i_{X} \omega=0$, il existe une $n-2$-forme $\kappa_{\lambda}$ qui dépend régulièrement du paramètre telle que $i_{X} \omega=\mathrm{d} Q \wedge \kappa_{\lambda}$. On obtient ainsi

$$
g_{1}^{*}(\omega)-\omega=\mathrm{d} Q \wedge \mathrm{d} \nu_{\lambda, g} .
$$


En reprenant l'argument de [G] et [CV-V], on fait une construction analogue pour tout les éléments $g$ de $K$. On moyennise alors par la mesure de Haar unitaire sur le groupe et on obtient une déconposition de la $n$-forme :

$$
\omega=B(x, \lambda) \mathrm{d} x_{1} \wedge \ldots \wedge \mathrm{d} x_{n}=\omega+\mathrm{d} Q \wedge \mathrm{d} \nu_{\lambda},
$$

où les formes $\omega$ et $\nu_{\lambda}$ sont $K$-invariantes et régulières dans leurs dépendances du paramètre puisque obtenues par moyennisation.

Troisième étape : cohomologie relative à paramètres

THÉORÈME 1.2. - Soit

$$
B(x, \lambda) \mathrm{d} x_{1} \wedge \ldots \wedge \mathrm{d} x_{n}
$$

une $n$-forme régulière $\left(C^{\infty}, C^{\omega}\right)$ qui dépend de façon régulière d'un paramètre, il existe une décomposition

$$
B(x, \lambda) \mathrm{d} x_{1} \wedge \ldots \wedge \mathrm{d} x_{n}=S(Q, \lambda) \mathrm{d} x_{1} \wedge \ldots \wedge \mathrm{d} x_{n}+\mathrm{d} Q \wedge \mathrm{d} n_{\lambda},
$$

où $S$ est une fonction régulière $\left(C^{\infty}, C^{\omega}\right)$ de $Q$ et de $\lambda$, et où la $n$-2-forme $n_{\lambda}$ dépend aussi de manière régulière $\left(C^{\infty}, C^{\omega}\right)$ du paramètre.

Preuve

i) Pour la signature $(p, q)$ avec $p>2, q>2$

Le théorème résulte dans ce cas de ce qui précède puisque une forme $K$-invariante est de la forme $S(Q, \lambda) \mathrm{d} x_{1} \wedge \ldots \wedge \mathrm{d} x_{n}$.

ii) Pour les signatures $(1, q)$ et $(p, 1)$

Les formes plates ne sont plus nécessairement de la forme cherchée. Il faut alors résoudre une équation cohomologique; étant donnée $h(x, \lambda)$ régulière et $X$ un champ hyperbolique, trouver $g(x, \lambda)$ telle que :

$$
X \cdot g(x, \lambda)=h(x, \lambda) .
$$

Cette question a été abordée en toute généralité par Roussarie dans [Ro] et dans le contexte particulier qui nous occupe ici, où le champ $X$ est linéaire, par [G] et [CV-V] lorsque les données sont sans paramètre. Comme la solution est donnée par une formule intégrale, il est clair que la dépendance régulière par rapport au paramètre subsiste. 
Quatrième étape : version à paramètre de la méthode des chemins

On applique alors à nouveau la méthode des chemins comme on le faisait en $[F]$ pour démontrer une extension du lemme de Morse isochore. II n'est pas nécessaire de repéter ici l'argument puisqu'il est identique, et on aboutit à l'existence d'un système de coordonnées $x$ dans lequel

$$
\omega=S(Q, \lambda) \mathrm{d} x_{1} \wedge \ldots \wedge \mathrm{d} x_{n} \text { et } f(x, \lambda)=Q .
$$

Cinquième étape : construction du changement de coordonnée isochore

On termine alors la démonstration du théorème 1.1 comme dans $[F]$. On fait un changement de coordonnées :

$$
x_{1}^{\prime}=x_{1} u(Q), \ldots, x_{n}^{\prime}=x_{n} u(Q),
$$

en sorte que

$$
S(u, \lambda)=u^{n-1}\left(u+Q \frac{\mathrm{d} u}{\mathrm{~d} Q}\right)
$$

La fonction $F(Q, \lambda)$ s'obtient alors en inversant $Q^{\prime}=Q u^{2}(Q)$ en $Q=$ $F\left(Q^{\prime}, \lambda\right)$. La dépendance régulière par rapport au paramètre se contrôle aisément.

2. Le lemme de Morse isochore transverse

Après la section 1, on peut supposer que la fonction $f$ est de la forme

$$
\begin{gathered}
f=F(Q, y)=Q(1+G(Q, y)) \\
\omega=\mathrm{d} x_{1} \wedge \ldots \wedge \mathrm{d} x_{n} .
\end{gathered}
$$

In existe donc des fonctions régulières $A_{i}(x, y)$ nulles en $(0,0)$ telles que

$$
f=\Sigma_{i} a_{i}(y) x_{i}^{2}\left(1+A_{i}(x, y)\right) .
$$

Terminons la démonstration du théorème annoncé.

Par le théorème des fonctions implicites, il existe des fonctions analytiques $y^{\prime}=y^{\prime}(x, y)$ tangentes à $y$ à l'ordre deux telles que

$$
\begin{gathered}
a_{i}\left(y^{\prime}\right)=a_{i}(y) x_{i}^{2}\left(1+A_{i}(x, y)\right) . \\
-87-
\end{gathered}
$$


On utilise, pour conclure le difféomorphisme local

$$
(x, y) \rightarrow\left(x^{\prime}, y^{\prime}(x, y)\right)
$$

Ce difféomorphisme conserve la forme volume

$$
\omega=\mathrm{d} x_{1} \wedge \ldots \wedge \mathrm{d} x_{n}
$$

et conjugue $f$ à $\Sigma_{i} a_{i}(y) x_{i}^{2}$.

Nous terminons cet article en donnant une application à l'étude des intégrales de la phase stationnaire.

En géométrie symplectique, un système hamiltonien sur un espace cotangent qui est une perturbation d'un flot géodésique s'écrit localement :

$$
H(x, y)=\Sigma_{i} a_{i}(y) x_{i}^{2}+R(x, y) .
$$

Notons $P=\Sigma_{i} a_{i}(y) x_{i}^{2}$.

On s'intéresse aux intégrales de la phase stationnaire :

$$
Z(\beta)=\int \exp (-\beta H(x, y)) \mathrm{d} x_{1} \wedge \ldots \wedge \mathrm{d} x_{n} \wedge \mathrm{d} y_{1} \wedge \ldots \wedge \mathrm{d} y_{n}
$$

Le théorème fournit une procédure qui permet de découper l'intégration en un intégration dans la base et une intégration suivant les variables de la fibre. On commence, en effet, par appliquer le lemme de Morse à paramètres à la fonction $H$ et on obtient une fonction

$$
P+c_{2}(y) P^{2}+c_{3}(y) P^{3}+\cdots+c_{k}(y) P^{k}+\ldots
$$

À l'issue de cette première étape, on trouve :

$Z(\beta)=\int \exp \left(-\beta\left(P+c_{2}(y) P^{2}+c_{3}(y) P^{3}+\cdots+c_{k}(y) P^{k}+\cdots\right)\right) \mathrm{d} x \wedge \mathrm{d} y$.

Comme d'usage dans le sujet, le théorème de Stokes permet toujours de se ramener à une intégrale à $P$ constant. (Pour fixer les idées, on peut supposer que l'ensemble $P \leq t$ est compact.) On comprend alors que le deuxième changement de variable que l'on fait dans la démonstration du théorème permet de se ramener à une intégrale sur les variables de la base $y$. 


\section{Références}

[A] ARnol'd (V.I.) . - Normal forms of Poisson structures and of other powers of volume forms, Tr. Sem. Im. Petrovskogo 12 (1985), pp. 1-15.

[CV-V] Colin DE Verdière (Y.) et VEY (J.) .- Le lemme de Morse isochore, Topology 18 (1979), pp. 283-293.

[F] Françoise (J.-P.) - - Modèle local simultané d'une fonction et d'une forme de volume, Astérisque 59-60 (1978), pp. 119-130.

[G] Guillemin (V.) . - Band asymptotics in two dimensions, Advances in Math. 42 (1981), pp. 248-282.

[G-S] Guillemin (V.) et Sternberg (S.) .- Symplectic technics in Physics, Cambridge University Press, Cambridge, 1983.

[K-L] Kostov (V.P.) et Lando (S.K.) . - Versal deformations of powers of volume forms, Prépublication 1992, Université de Nice.

[L] Lando (S.K.) .- Normal forms of the degrees of a volume form, Funct. Anal. and its Applications 19, $\mathrm{n}^{\circ} 2$ (1985), pp. 146-148.

[R] Roche (C.) .- Cohomologie relative dans le domaine réel, Thèse de Doctorat, Université de Grenoble (1982).

[Ro] Roussarie (R.) . - Modèles locaux de champs et de formes, Astérisque 30 (1979).

[V.] VARCHENKo (A.) . - Local classification of volume forms in the presence of a hypersurface, Funct. Anal. and its Applications 19, $\mathrm{n}^{\circ} 4$ (1985), pp. 23-31.

[V] VeY (J.) . - Sur le lemme de Morse, Inventiones Mat. 40 (1977), pp. 1-10. 